\title{
Comunicación
}

\section{Niveles de Calcio y Fósforo Sérico en Guacamayos (Ara sp) Mantenidos en Cautiverio en Lima, Perú}

\author{
Calcium and Phosphorus Serum Levels in Macaws (Ara sp) Kept in \\ Captivity in Lima, Peru
}

\author{
Nancy Carlos Erazo ${ }^{1,2}$, Noemí Coronel Defilippi ${ }^{2}$
}

\section{Resumen}

El objetivo del estudio fue determinar los valores de calcio y fósforo sérico en guacamayos (Ara sp) mantenidos en cautiverio en tres zoológicos del departamento de Lima, Perú. Se trabajó con la totalidad de la población de cada centro, siendo 34 aves adultas distribuidas en 4 especies (9 Ara macao, 14 Ara ararauna, 4 Ara chlropterus y 7 Ara severus). Se recolectó $0.8 \mathrm{ml}$ se sangre de la vena yugular de cada ave en tubos al vacío sin anticoagulante. Los niveles séricos de calcio y fósforo fueron determinados por un método colorimétrico. Los valores promedio de calcio fueron de $9.69 \pm 1.48 \mathrm{mg} / \mathrm{dl}$ y de fósforo fueron de $6.08 \pm 1.42$ $\mathrm{mg} / \mathrm{dl}$. Por especie, los valores de calcio fueron: A. ararauna $9.67 \pm 1.68 \mathrm{mg} / \mathrm{dl}, A$. chloropterus $9.47 \pm 1.80 \mathrm{mg} / \mathrm{dl}$, $A$. macao $9.90 \pm 1.75 \mathrm{mg} / \mathrm{dl}$ y $A$. severus $9.60 \pm 0.35$; y para fósforo fueron: A. ararauna $6.03 \pm 1.45 \mathrm{mg} / \mathrm{dl}$, A. chloropterus $7.05 \pm 2.12$ $\mathrm{mg} / \mathrm{dl}$, A. macao $6.14 \pm 1.35 \mathrm{mg} / \mathrm{dl}$ y $A$. severus $5.54 \pm 0.87 \mathrm{mg} / \mathrm{dl}$. Se observaron diferencias significativas de los valores de calcio y fósforo según el lugar de procedencia de las aves.

Palabras clave: calcio, cautiverio, fósforo, guacamayos, minerales, psitácidos

\section{AbSTRACT}

The aim of the study was to determine the values of serum calcium and phosphorus in macaws (Ara sp) kept in captivity in three zoos in the department of Lima, Perú. All available births were sampled. There were 34 adult birds in 4 species

${ }^{1}$ Programa de Ecología de Enfermedades y Medicina de la Conservación, Centro de Ornitología y Biodiversidad (CORBIDI), Lima, Perú

${ }^{2}$ Escuela Académica Profesional de Ciencias Agropecuarias, Facultad de Medicina Veterinaria, Universidad Alas Peruanas, Lima, Perú

${ }^{3}$ E-mail: nancy.carlos.erazo@gmail.com

Recibido: 23 de enero de 2016

Aceptado para publicación: 28 de abril de 2016 
(9 Ara macao, 14 Ara ararauna, 4 Ara chlropterus and 7 Ara severus). Blood samples $(0.8 \mathrm{ml})$ were taken from the jugular vein in vacuum tubes without anticoagulant. Serum calcium and phosphorus values were determined by a colorimetric method. The mean values of calcium was $9.70 \pm 1.48 \mathrm{mg} / \mathrm{dl}$ and phosphorus was $6.08 \pm 1.42 \mathrm{mg} / \mathrm{dl}$. By species, calcium values were: A ararauna $9.67 \pm 1.68 \mathrm{mg} / \mathrm{dl}$, A chloropterus $9.47 \pm 1.80$ $\mathrm{mg} / \mathrm{dl}$, A. macao $9.90 \pm 1.75 \mathrm{mg} / \mathrm{dl}$, A. severus $9.60 \pm 0.35 \mathrm{mg} / \mathrm{dl}$, and for phosphorus were: A. ararauna $6.03 \pm 1.45 \mathrm{mg} / \mathrm{dl}$, A. chloropterus $7.05 \pm 2.12 \mathrm{mg} / \mathrm{dl}$, A. macao $6.14 \pm 1.35 \mathrm{mg} /$ $\mathrm{dl}$, A. severus $5.54 \pm 0.87 \mathrm{mg} / \mathrm{dl}$. Significant differences in the levels of calcium and phosphorus were observed among the origin of birds.

Key words: calcium, captivity, phosphorus, macaws, minerals, Psittacidae

\section{INTRODUCCIÓN}

Los guacamayos son aves del género Ara y pertenecen a la familia Psittacidae. Son individuos muy vistosos y cumplen un rol ecológico importante al ser dispensadores de semillas (Jordán, 2009). Son aves susceptibles al tráfico de fauna silvestre para ser comercializadas mayormente como «mascotas», predisponiéndolas a un inadecuado manejo y presentación de innumerables enfermedades.

El calcio y el fósforo son minerales relacionados en su metabolismo e indispensables para la salud y el correcto funcionamiento del organismo; actuando en el funcionamiento del esqueleto y músculos, puesta de huevos, contracción del corazón y digestión, entre otros. Las deficiencias de estos minerales pueden afectar el desarrollo del esqueleto y predisponer a diversas patologías (Mora, 1991).

La dieta ofrecida a aves silvestres en cautiverio puede resultar deficiente o inadecuada, desencadenando enfermedades metabólicas como hipocalcemia, osteomalacia y raquitismo (Jordán, 2009; Soto y Bert, 2011). Estos desórdenes metabólicos pueden presentarse con signos clínicos poco específicos o en forma subclínica, siendo necesaria la ayuda de pruebas complementarias de laboratorio, como por ejemplo, para determinar los valores séricos de calcio y fósforo (Samour, 2010). Valores referenciales de es- tos minerales en guacamayos han sido reportados a nivel internacional (Lumeij y Oberduim., 1990; ISIS, 2002; De Faria et al., 2008; Jiménez et al., 2009; Meredith y Redrobe, 2009). Una de las principales referencias internacionales está dada por el Sistema Internacional de Información de Especies (ISIS, del inglés International Species Information System), aunque muchos de estos reportes provienen de pocos animales, sin especificación de edad, sexo o tipo de alimentación, entre otros.

Debido a la importancia fisiológica del calcio y fósforo para el estado de salud de los psitácidos, el objetivo del presente estudio fue determinar los niveles de calcio y fósforo sérico en guacamayos mantenidos en cautiverio en el departamento de Lima. Con estos valores, se podrá disponer de parámetros locales de referencia dentro de un manejo y dieta más cercana a la realidad del país a fin de apoyar el diagnóstico y tratamiento oportuno de diversas enfermedades en este tipo de aves y apoyar la conservación de estas especies.

\section{Materiales y Métodos}

El estudio se llevó a cabo en tres zoológicos de dos provincias del departamento de Lima, Perú, durante el mes de abril de 2015: «La Granja Villa y su Mundo Mágico» y «La Granja Villa Norte y su mundo Mágico», 
Cuadro 1. Valores de calcio y fosforo sérico (promedio \pm D.E.) por especies de guacamayos ( $A r a \mathrm{sp}$ ) mantenidos en cautiverio en el departamento de Lima

\begin{tabular}{lccccccccc}
\hline & & \multicolumn{4}{c}{$\begin{array}{c}\text { Calcio } \\
(\mathrm{mg} / \mathrm{dl})\end{array}$} & \multicolumn{4}{c}{$\begin{array}{c}\text { Fosforo } \\
(\mathrm{mg} / \mathrm{dl})\end{array}$} \\
\cline { 2 - 10 } & & Prom. & $\mathrm{DS}$ & Min & Max & Prom. & DS & Min & Max \\
\hline Ara ararauna & 14 & 9.67 & 1.68 & 6.04 & 12.10 & 6.03 & 1.45 & 3.91 & 9.00 \\
Ara chloropterus & 4 & 9.47 & 1.80 & 8.21 & 12.10 & 7.05 & 2.12 & 4.23 & 9.00 \\
Ara macao & 9 & 9.90 & 1.75 & 8.27 & 13.80 & 6.14 & 1.35 & 3.53 & 7.89 \\
Ara severus & 7 & 9.60 & 0.35 & 9.08 & 10.05 & 5.54 & 0.87 & 4.13 & 6.60 \\
\hline Ara $\mathrm{sp}$ & 34 & 9.70 & 1.48 & 6.04 & 13.80 & 608 & 1.42 & 3.53 & 9.00 \\
\hline
\end{tabular}

ubicados en la provincia de Lima, $\mathrm{y}$ «Warmi», en la provincia de Huaral.

Para el estudio se tomó la totalidad de población de guacamayos albergados en los tres zoológicos, correspondiendo a 34 individuos adultos de sexo indeterminado: 14 guacamayos azul y amarillo (Ara ararauna), 4 guacamayos rojo y verde (Ara chloropterus), 9 guacamayos escarlata (Ara macao) y 7 guacamayos frente castaña (Ara severus).

Los animales fueron colocados en posición latero-lateral para obtener muestras de sangre $(0.8 \mathrm{ml})$ de la vena yugular. Las muestras fueron recolectadas en tubos al vacío sin anticoagulante y conservadas a $4{ }^{\circ} \mathrm{C}$ hasta obtener el suero. Las muestras fueron remitidas al laboratorio para el análisis de calcio y fósforo utilizando la espectrofotometría, mediante el método colorimétrico cuantitativo para determinar los niveles totales de calcio y fósforo sérico. Para la determinación de calcio se utilizó el kit Stanbio Total Calcium LiquiColor ${ }^{\circledR}$ (Arsenazo III) con el reactivo Cat. N. ${ }^{\circ} 0156$ (Stanbio Laboratory). Para la determinación de fósforo se utilizó el Kit Stanbio Phosphorus Liqui-UV® con el reactivo Cat. N. ${ }^{\circ} 0831$ (Stanbio Laboratory).
Se empleó estadística descriptiva (promedios y desviación estándar) de cada parámetro y por especie. Además, se determinaron posibles diferencias según lugar de procedencia del ave mediante análisis de varianza, con un nivel de un nivel de confianza del 95\% $(\mathrm{p}<0.05)$ utilizando el programa estadístico SPSS® v. 212012.

\section{Resultados}

En el Cuadro 1 se muestran los valores de calcio y fósforo obtenidos para la población total del estudio y en los cuadros 2 y 3 se presentan los valores por especie para cada zoológico. Se obtuvieron diferencias significativas para el valor de calcio entre el zoológico «La Granja Villa y su Mundo Mágico» y «Warmi» $(\mathrm{p}=0.0016)$, y para el valor de fósforo entre «La Granja Villa y su Mundo Mágico» $\mathrm{y}$ «Warmi» $(\mathrm{p}=0.0031)$, así como con «La Granja Villa Norte» $(\mathrm{p}=0.004)$.

\section{Discusión}

Según la bibliografía encontrada, el presente estudio es el primer reporte de los valores de calcio y fósforo sérico en guaca- 
Cuadro 2. Valores de calcio sérico (mg/dl, promedio \pm D.E) en guacamayos (Ara $\mathrm{sp}$ ) mantenidos en cautiverio en tres zoológicos del departamento de Lima

\begin{tabular}{lccccccccc}
\hline & \multicolumn{3}{c}{ "Warmi" } & \multicolumn{3}{c}{$\begin{array}{c}\text { "La Granja Villa y su } \\
\text { mundo mágico" }\end{array}$} & \multicolumn{3}{c}{ "La Granja Villa } \\
& \multicolumn{3}{c}{} & \multicolumn{4}{c}{ Norte" } \\
\cline { 2 - 11 } & $\mathrm{n}$ & Prom & DS & $\mathrm{n}$ & Prom. & DS & $\mathrm{n}$ & Prom. & DS \\
\hline Ara ararauna & 8 & 8.48 & 1.09 & 2 & 11.75 & 0.49 & 4 & 11.02 & 0.51 \\
Ara chloropterus & 2 & 8.31 & 0.13 & 2 & 10.65 & 2.05 & 0 & - & - \\
Ara macao & 4 & 9.58 & 1.34 & 2 & 11.95 & 2.62 & 3 & 8.97 & 0.62 \\
Ara severus & 2 & 9.43 & 0.49 & 2 & 9.39 & 0.16 & 3 & 9.87 & 0.21 \\
\hline Ara sp & 16 & $8.85^{\mathrm{a}}$ & 1.11 & 8 & $10.93^{\mathrm{b}}$ & 1.00 & 10 & $10.06^{\mathrm{ab}}$ & 1.68 \\
\hline
\end{tabular}

${ }^{a, b}$ Superíndices diferencias entre promedios totales indican dife rencia esta dística $(p<0.05)$

Cuadro 3. Valores de fósforo sérico ( $\mathrm{mg} / \mathrm{dl}$, promedio \pm D.E) en guacamayos (Ara $\mathrm{sp}$ ) mantenidos en cautiverio en tres zoológicos del departamento de Lima

\begin{tabular}{lccccccccc}
\hline & \multicolumn{3}{c}{ "Warmi" } & \multicolumn{3}{c}{$\begin{array}{c}\text { La Granja Villa y su } \\
\text { mundo mágico" }\end{array}$} & \multicolumn{3}{c}{$\begin{array}{c}\text { La Granja Villa } \\
\text { Norte" }\end{array}$} \\
\cline { 2 - 11 } & $\mathrm{n}$ & Prom & DS & $\mathrm{n}$ & Prom. & DS & $\mathrm{n}$ & Prom. & DS \\
\hline Ara ararauna & 8 & 5.30 & 1.04 & 2 & 8.75 & 0.11 & 4 & 6.12 & 0.36 \\
Ara chloropterus & 2 & 5.46 & 1.73 & 2 & 8.65 & 0.50 & 0 & - & - \\
Ara macao & 4 & 6.73 & 1.24 & 2 & 6.75 & 0.64 & 3 & 4.93 & 1.22 \\
Ara severus & 2 & 6.06 & 0.48 & 2 & 6.15 & 0.64 & 3 & 4.78 & 0.65 \\
\hline Ara $\mathrm{sp}$ & 16 & $5.77^{\mathrm{a}}$ & 1.19 & 8 & $7.56^{\mathrm{b}}$ & 0.93 & 10 & $5.36^{\mathrm{a}}$ & 1.29 \\
\hline
\end{tabular}

${ }^{a, b}$ Superíndices diferencias entre promedios totales indican diferencia estadística $(p<0.05)$

mayos (Ara sp) en el Perú. Jiménez et al. (2009) reportan amplios valores de calcio (8.5$13.0 \mathrm{mg} / \mathrm{dl})$ y fosforo $(2.0-10 \mathrm{mg} / \mathrm{dl})$ para el género Ara. Sin embargo, Lumeij y Oberduim (1990) reportan valores mucho menores de calcio $(2.3 \pm 0.2 \mathrm{mg} / \mathrm{dl})$, pero la diferencia podría deberse a los materiales y métodos utilizados. Además, se debe considerar que bajos niveles de albúmina, como lo reportado en loros, podrían ocasionar niveles bajos de calcio (Lumeij y Overduim, 1990; Yosted y Carrascosa, 2011).

Para el guacamayo azul y amarillo ( $A$. ararauna), los valores de Ca y $\mathrm{P}$ se encontraron dentro de los rangos brindados por Meredith y Redrobe (2012) (Ca 8.8-11.2 mg/ dl), De Faria et al. (2008) (Ca 9.1-9.8 mg/dl 
y P 4.9 a $6.4 \mathrm{md} / \mathrm{dl})$ e ISIS (2002) (Ca 6.0$15 \mathrm{mg} / \mathrm{dl}$ y P $1.0-14.0 \mathrm{mg} / \mathrm{dl})$. Tomando como referencia el valor promedio y rangos reportados en ISIS (2002), los valores de Ca y P del estudio fueron similares para el guacamayo rojo y verde (A. chloropterus) (Ca 9.10 $\pm 1.0 \mathrm{mg} / \mathrm{dl}$ y P $1.2-9.3 \mathrm{mg} / \mathrm{dl})$, el guacamayo escarlata (A. macao) $(\mathrm{Ca} 9.90 \pm 1.75 \mathrm{mg} / \mathrm{dl}$ y P $1.0-14.0 \mathrm{mg} / \mathrm{dl})$ y el guacamayo de frente castaña $($ A. severus $)(\mathrm{Ca} 6.9-12 \mathrm{mg} / \mathrm{dl})$.

Los resultados obtenidos indican que los niveles Ca y P sérico para los individuos del estudio son adecuados, a pesar que estos tendrían una dieta y manejo diferente en comparación con otros zoológicos a nivel internacional. Sin embargo, el valor de fósforo para $A$. severus se encontró ligeramente por encima del rango brindado por ISIS (2002) (1.5$5.3 \mathrm{mg} / \mathrm{dl}$ ), aunque sin aparente importancia clínica, ya que no se observaron signos clínicos de hiperfosfatemia, como calcificación de tejidos blandos en la córnea y piel (Vaca, 2003). Además, los niveles de fósforo sérico varían según la dieta, función hormonal y función renal de los individuos (Sodikof, 1996).

Por último, las diferencias encontradas entre zoológicos se deberían a la dieta y suplementos ofrecidos. «Warmi» no adiciona suplementos vitamínicos y minerales que podrían explicar el valor de Ca y $\mathrm{P}$ hallado, mientras que «La Granja Villa y su Mundo Mágico» proporciona abundante cantidad de menestras, alimentos que son conocidos por su aporte de fósforo.

\section{Conclusiones}

- Se determinaron los valores de calcio y fósforo para el género Ara y cuatro especies de guacamayos (A. ararauna, $A$. chloropterus, A. macao y A. severus) en cautiverio.

- Se observaron diferencias estadísticas de los valores de calcio y fósforo según el zoológico de procedencia.

\section{Literatura Citada}

1. De Faria S, Da Costa M, Alves R, Gil L, Capacchi N, Franca R, Locatello M. 2008. Parâmetros de bioquímica sérica de machos, fêmeas e filhotes de Araras canindé (Ara ararauna) saudáveis mantidas em cativeiro comercial. Cienc Rural 38: 711-716. doi: 10.1590/S0103-84782008000300018

2. [ISIS] International Species Information System. 2002. Reference ranges for physiological data values of Ara spp, USA. [Internet]. Disponible en: http://www.isis.org.com.

3. Lumeij J, Overduim. 1990. Plasma chemistry references values in Psittaciformes. Avian Pathol 19: 235-244. doi: 10.1080/03079459008418676

4. Jiménez J, Domingo R, Crosta L, Domingo R. 2009. Manual clínico de animales exóticos. España: Multimédica. $209 \mathrm{p}$.

5. Jordán R. 2009. Guacamayos: una guía completa. España: Hispano Europea. 150 p.

6. Meredith A, Redrobe S. 2012. Manual de animales exóticos. $4^{\mathrm{a}}$ ed. Madrid: BSVA. $434 \mathrm{p}$.

7. Mora Y. 1991. Nutrición animal. Costa Rica: Ed Universidad Estatal a Distancia. $120 \mathrm{p}$.

8. Samour J. 2010. Medicina aviaria. $2^{\text {a }}$ ed. Barcelona, España: Elsevier Mosby. $544 \mathrm{p}$.

9. Sodikof S. 1996. Pruebas diagnósticas y de laboratorio en las enfermedades de pequeños animales. España: Mosby. 315 p.

10. Soto C, Bert E. 2011. Principios en la alimentación de psitácidas (Torino-Italia). REDVET 12(11). [Internet]. Disponible en: www.veterinaria.org/revistas/redvet/ n111111/111110.pdf

11. Vaca L. 2003. Producción avícola. Costa Rica: Ed Universidad Estatal a Distancia. $357 \mathrm{p}$.

12. Yosted D, Carrascosa A. 2011. Patología del metabolismo del calcio. Protoc Diagn Ter Pediat 1: 177-192. 